\title{
Welche Mehrheiten brauchen Fiskalpakt und ESM?
}

VB

verfassungsblog.de/welche-mehrheiten-brauchen-fiskalpakt-und-esm/

Hanno Kube Do 5 Apr 2012

Do $5 \mathrm{Apr}$

2012

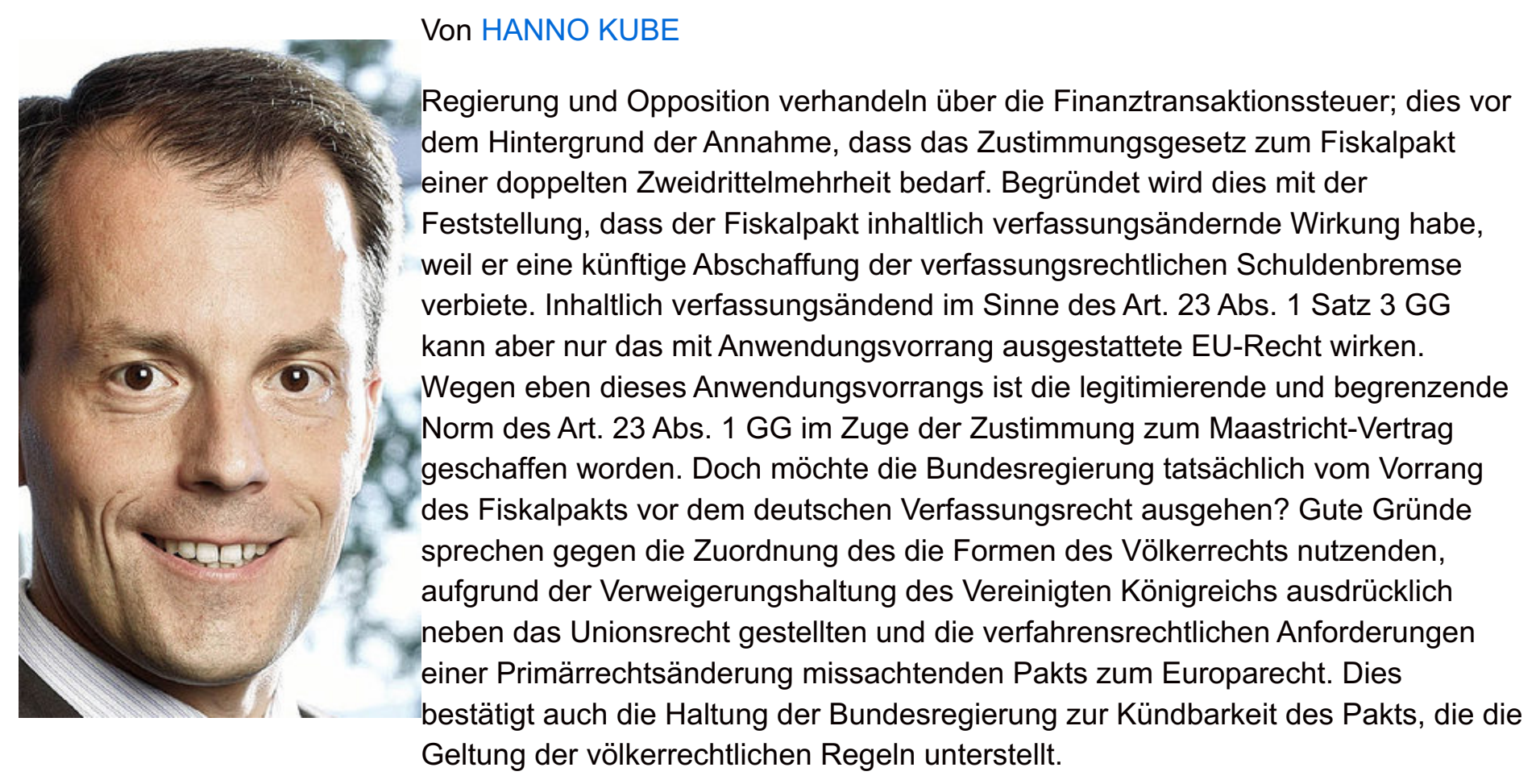

Als völkerrechtlicher Vertrag unterfällt der Fiskalpakt deshalb allein Art. 59 Abs. 2 GG. Zustimmungsgesetze zu völkerrechtlichen Verträgen bedürfen danach im Grundsatz allein der einfachen Parlamentsmehrheit. Die Bundesrepublik hat sich auf dieser Grundlage seit Jahrzehnten völkerrechtlich gebunden und dabei auch Hoheitsrechte übertragen (siehe auch Art. 24 Abs. 1 GG). Allenfalls wäre zu erwägen, mit Blick auf den Sinn und Zweck von Art. 59 Abs. 2 GG zu verlangen, die Zustimmung zu einer völkerrechtlichen Verpflichtung, das Verfassungsrecht zu ändern, in der Form eines entsprechend verfassungsändernden Gesetzes zu erteilen. Doch müsste dies ein verfassungstextänderndes Gesetz sein, wie es Art. 79 Abs. 1 GG verlangt. Eine Textänderung sieht der aktuelle Entwurf aber nicht vor.

Konsequent ist dies freilich insoweit, als die Regierung - was wegen der Nichteinbeziehung der Verschuldung durch die mittelbare Staatsverwaltung allerdings fraglich ist - davon ausgeht, mit Art. 109 Abs. 3, Art. 115 Abs. 2 GG bereits alles Erforderliche geregelt zu haben. Verfassungsändend soll die Pflicht wirken, die Schuldenbremse erhalten zu müssen. Die Erfüllung einer solchen Pflicht lässt sich aber normativ kaum innerstaatlich sichern. Zu denken wäre allenfalls an eine Ergänzung von Art. 79 Abs. 3 GG (durch die verfassunggebende Gewalt?). Doch könnte auch eine solche Ergänzung jederzeit wieder rückgängig gemacht werden. Die normativ verlässliche Sicherung der künftigen Nichtänderung von Recht ist aussichtslos und sollte nicht erstrebt werden. Und sie muss bei genauer Betrachtung auch gar nicht erstrebt werden. Denn völkerrechtlich geschuldet ist das Bestehen der Schuldenbremse, nicht die Verhinderung ihrer Abschaffung. Die Zustimmung zum Fiskalpakt bedarf mithin der einfachen Mehrheit, nicht weniger, aber auch nicht mehr.

Verwunderlich ist es im Angesicht der regierungsseitigen Einordnung des Fiskalpakts freilich, dass die Zustimmung zum ESM seinerseits allein der einfachen Mehrheit bedürfen soll. Denn der ESM steht, gerade aufgrund der Rückbindung durch den neuen Art. 136 Abs. 3 AEUV, dem Europarecht und dem ihm eigenen Anwendungsvorrang formal und auch inhaltlich sehr viel näher als der Fiskalpakt. Die Auswirkungen auf die Wahrnehmung des Budgetrechts nach Art. 110 GG und auf die Einhaltbarkeit der Verschuldungsgrenzen nach Art. 109 Abs. 3, Art. 115 
Abs. 2 GG sind deutlich. Die Rechtsfolgen des Art. 23 Abs. 1 Satz 3 GG (Erfordernis der doppelten Zweidrittelmehrheit, einhergehender Rückzug der verfassungsrechtlichen Prüfung auf die Grenzen des Art. 79 Abs. $3 \mathrm{GG}$ ) werden daher im Fall des ESM, nicht im Fall des Fiskalpakts ausgelöst.

So hat die Opposition durchaus eine Verhandlungsposition; nicht aber diejenige, von der verbreitet ausgegangen wird.

Hanno Kube ist Inhaber des Lehrstuhls für Öffentliches Recht und Steuerrecht an der Universität Mainz.

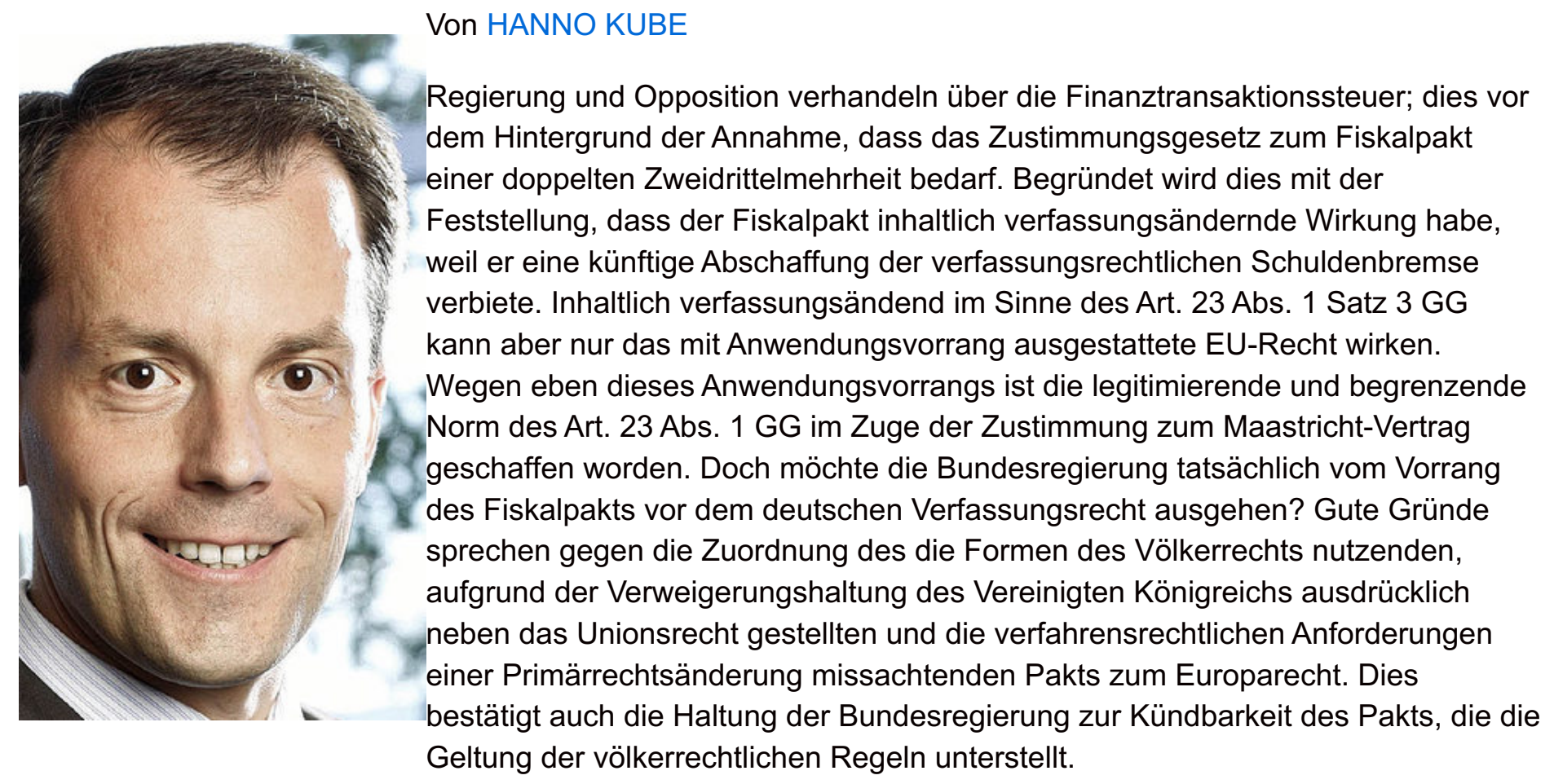

Als völkerrechtlicher Vertrag unterfällt der Fiskalpakt deshalb allein Art. 59 Abs. 2 GG. Zustimmungsgesetze zu völkerrechtlichen Verträgen bedürfen danach im Grundsatz allein der einfachen Parlamentsmehrheit. Die Bundesrepublik hat sich auf dieser Grundlage seit Jahrzehnten völkerrechtlich gebunden und dabei auch Hoheitsrechte übertragen (siehe auch Art. 24 Abs. 1 GG). Allenfalls wäre zu erwägen, mit Blick auf den Sinn und Zweck von Art. 59 Abs. 2 GG zu verlangen, die Zustimmung zu einer völkerrechtlichen Verpflichtung, das Verfassungsrecht zu ändern, in der Form eines entsprechend verfassungsändernden Gesetzes zu erteilen. Doch müsste dies ein verfassungstextänderndes Gesetz sein, wie es Art. 79 Abs. 1 GG verlangt. Eine Textänderung sieht der aktuelle Entwurf aber nicht vor.

Konsequent ist dies freilich insoweit, als die Regierung - was wegen der Nichteinbeziehung der Verschuldung durch die mittelbare Staatsverwaltung allerdings fraglich ist - davon ausgeht, mit Art. 109 Abs. 3, Art. 115 Abs. 2 GG bereits alles Erforderliche geregelt zu haben. Verfassungsändend soll die Pflicht wirken, die Schuldenbremse erhalten zu müssen. Die Erfüllung einer solchen Pflicht lässt sich aber normativ kaum innerstaatlich sichern. Zu denken wäre allenfalls an eine Ergänzung von Art. 79 Abs. 3 GG (durch die verfassunggebende Gewalt?). Doch könnte auch eine solche Ergänzung jederzeit wieder rückgängig gemacht werden. Die normativ verlässliche Sicherung der künftigen Nichtänderung von Recht ist aussichtslos und sollte nicht erstrebt werden. Und sie muss bei genauer Betrachtung auch gar nicht erstrebt werden. Denn völkerrechtlich geschuldet ist das Bestehen der Schuldenbremse, nicht die Verhinderung ihrer Abschaffung. Die Zustimmung zum Fiskalpakt bedarf mithin der einfachen Mehrheit, nicht weniger, aber auch nicht mehr.

Verwunderlich ist es im Angesicht der regierungsseitigen Einordnung des Fiskalpakts freilich, dass die Zustimmung zum ESM seinerseits allein der einfachen Mehrheit bedürfen soll. Denn der ESM steht, gerade aufgrund der Rückbindung durch den neuen Art. 136 Abs. 3 AEUV, dem Europarecht und dem ihm eigenen Anwendungsvorrang 
formal und auch inhaltlich sehr viel näher als der Fiskalpakt. Die Auswirkungen auf die Wahrnehmung des Budgetrechts nach Art. 110 GG und auf die Einhaltbarkeit der Verschuldungsgrenzen nach Art. 109 Abs. 3, Art. 115 Abs. 2 GG sind deutlich. Die Rechtsfolgen des Art. 23 Abs. 1 Satz 3 GG (Erfordernis der doppelten Zweidrittelmehrheit, einhergehender Rückzug der verfassungsrechtlichen Prüfung auf die Grenzen des Art. 79 Abs. $3 \mathrm{GG}$ ) werden daher im Fall des ESM, nicht im Fall des Fiskalpakts ausgelöst.

So hat die Opposition durchaus eine Verhandlungsposition; nicht aber diejenige, von der verbreitet ausgegangen wird.

Hanno Kube ist Inhaber des Lehrstuhls für Öffentliches Recht und Steuerrecht an der Universität Mainz.

LICENSED UNDER CC BY NC ND

SUGGESTED CITATION Kube, Hanno: Welche Mehrheiten brauchen Fiskalpakt und ESM?, VerfBlog, 2012/4/05, http://verfassungsblog.de/welche-mehrheiten-brauchen-fiskalpakt-und-esm/. 